\title{
SOBRE LA CONVENIENCIA DE LA ADHESIÓN DEL PERÚ A LA CONVENCIÓN SOBRE EL DERECHO DE LOS USOS DE LOS CURSOS DE AGUA INTERNACIONALES PARA FINES DISTINTOS DE LA NAVEGACIÓN
}

REFLEXIONES DESDE EL DERECHO INTERNACIONAL PÚBLICO

\author{
Jorge Raffo Carbajal \\ Ministerio de Relaciones Exteriores \\ jraffo@rree.gob.pe \\ Pablo Rosales Zamora** \\ Pontificia Universidad Católica del Perú \\ pablo.rosalesz@pucp.pe \\ Diana Aduviri Choque \\ Pontificia Universidad Católica del Perú \\ diana.aduviri@gmail.com
}

Fecha de recepción: agosto de 2017 Fecha de aceptación: diciembre de 2017

* Jorge Raffo Carbajal es embajador en el Servicio Diplomático de la República. Se
desempeńa como director general de Tratados del Ministerio de Relaciones Exteriores
y jefe encargado de la Oficina General de Asuntos Legales. Es magíster en Comercio
Internacional y magíster en Gestión y Finanzas Internacionales por la Université Libre
de Bruxelles. Realizó estudios escolares en el Colegio San Agustín de Lima, estudios de
pregrado en la PUCP y estudios de postgrado en la Academia Diplomática del Perú, en el
Instituto Internacional de Administración Pública/Escuela Nacional de Administración
de París, en la Escuela de Comercio 'Solvay' de la Universidad Libre de Bruselas, en la
Facultad de Ciencias Políticas y Relaciones Internacionales de la Universidad Libre de 
RESUMEN: El presente artículo tiene como finalidad analizar los aspectos más relevantes de la Convención sobre el Derecho de los Usos de los Cursos de Agua Internacionales para Fines Distintos de la Navegación de las Naciones Unidas (1997). En este marco, el artículo presenta algunas nociones básicas del Derecho Internacional de Aguas, y la historia referida al surgimiento de esta Convención. En una segunda sección, se analiza, de manera detallada, qué principios y normas alberga la Convención. Por último, se profundiza sobre algunas razones de índole jurídica para la adhesión del Perú a esta Convención.

Bruselas y estudios en la Escuela de Gobierno de la Universidad San Martín de Porres. Actualmente es docente del curso de Gestión Pública en la Academia Diplomática del Perú desde el año 2014, habiendo ejercido la docencia en las universidades ecuatorianas Casagrande y Espíritu Santo en la cátedra de Globalización, Organismos Internacionales y Negociación. Durante su trayectoria diplomática en el exterior ha trabajado en Bélgica, Luxemburgo, Estados Unidos y Ecuador, desempeńándose en este último puesto como cónsul general. En la cancillería peruana ha trabajado como director general de Administración, director de Políticas Sociales de la Dirección General de Asuntos Multilaterales, jefe de gabinete del secretario general de Relaciones Exteriores, jefe del Departamento Argentina, Paraguay y Uruguay de la Dirección General de Asuntos Bilaterales, entre otros puestos.

* Pablo Rosales Zamora es magíster en Ciencia Política y Gobierno con mención en Relaciones Internacionales por la Escuela de Gobierno y Políticas Públicas de la Pontificia Universidad Católica del Perú (PUCP). Es también abogado por la misma casa de estudios y asesor legal de la Oficina de Derecho Internacional Público del Ministerio de Relaciones Exteriores (MRE) del Perú.

*** Diana Aduviri Choque es bachiller en Derecho por la Pontificia Universidad Católica del Perú. Es, además, integrante de la Oficina de Derecho Internacional Público del MRE. Asimismo, es investigadora del Círculo de Estudios Chinos en Derecho del Instituto Confucio PUCP (CECH) y asistente de docencia en el curso de Derecho del Comercio Internacional en la Facultad de Derecho de la PUCP. 
SOBRE LA CONVENIENCIA DE LA ADHESIÓN DEL PERÚ A LA CONVENCIÓN SOBRE EL

DERECHO DE LOS USOS DE LOS CURSOS DE AGUA INTERNACIONALES PARA FINES

DISTINTOS DE LA NAVEGACIÓN

Palabras Clave: Derecho Internacional de Aguas (DIA), Convención sobre el Derecho de los Usos de los Cursos de Agua Internacionales para Fines Distintos de la Navegación (UNWC), tratado, adhesión del Perú.

\section{ON THE DESIREBALITY OF THE ACCESSION OF PERU TO THE CONVENTION OF THE UNITED NATIONS ON THE LAW OF THE NON-NAVIGATIONAL USES OF INTERNATIONAL WATERCOURSES}

\section{Considerations from Public International LaW}

ABSTRACT: The present article aims to analyze the most relevant aspects of the Convention of the United Nations on the Law of the Non-navigational Uses of International Watercourses of 1997. In this framework, the article presents some basic notions of International Water Law and the history of the Convention's origins. In a second section, the article analyzes in detail what principles and norms are established by the Convention. Finally, the article furthers on some juridical reasons for the accession of Peru to this Convention.

KeYwords: International Water Law, Convention of the United Nations on the Law of the Non-navigational Uses of International Watercourses (UNWC), Treaty, Accession of Peru. 


\section{Introducción}

\subsection{Conceptos iniciales}

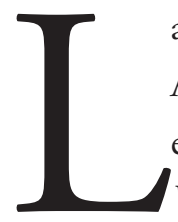

a Convención sobre el Derecho de los Usos de los Cursos de Agua Internacionales para Fines Distintos de la Navegación se enmarca en el Derecho Internacional de Aguas (International Water $L a w)^{1}$. Empero, es necesario conocer con mayor precisión algunos conceptos iniciales que conforman el significado de las denominadas aguas, entre los que están comprendidos el agua dulce, las aguas subterráneas o acuiferos, las cuencas de captación o cuencas hidrográficas y el curso de agua.

De esta forma, el punto de partida será el agua dulce, que representa menos del $2.5 \%$ del total de agua en el planeta. A su vez, esta puede clasificarse en agua superficial — conformada por ríos, lagos, humedales, y manantiales - o en agua por debajo de la superficie - también denominada agua subterránea o acuífero- (Autoridad Nacional del Agua, 2017). Sobre esto último, las aguas subterráneas o acuíferos, son unidades geológicas subterráneas de las cuales es posible extraer agua, por lo que "el acuífero alude tanto al agua subterránea como al espacio que lo contiene" (Autoridad Nacional del Agua, 2017, p. 17).

Siguiendo esta línea, es importante resaltar la existencia de un concepto general que abarca a otros conceptos mencionados, que es el de las cuencas de captación o cuencas hidrográficas. Estas se definen como una unidad geográfica e hidrológica, que comprende tanto a las aguas superficiales como a las subterráneas (acuíferos). Esta, igualmente, tiene como elemento central a su río principal y se conforma, además, "por todos los territorios comprendidos entre la naciente y la desembocadura

1 En adelante, la DIA. 


\section{SOBRE LA CONVENIENCIA DE LA ADHESIÓN DEL PERÚ A LA CONVENCIÓN SOBRE EL DERECHO DE LOS USOS DE LOS CURSOS DE AGUA INTERNACIONALES PARA FINES DISTINTOS DE LA NAVEGACIÓN}

de ese río (...) [incluyendo] todas las tierras y ríos menores que aportan agua a ese río principal (...)” (Aguilar \& Iza, 2009, pp. 9-10). Así, este sistema de arroyos y ríos converge en la misma desembocadura.

Por otra parte, el concepto curso de $a g u a^{2}$ no es un concepto integral que abarque un área geográfica completa, como sí lo es la noción de cuenca hidrográfica. Por el contrario, solamente comprende a las aguas superficiales y subterráneas que constituyen un conjunto unitario y que fluyen a una desembocadura común, siendo este último un concepto mucho más limitado (Aguilar \& Iza, 2009, p. 15). En adición, las cuencas hidrográficas, los cursos de agua, los ríos, entre otros, pueden tener carácter internacional si se extienden por el territorio de dos o más Estados. En consecuencia, estos pueden recibir el nombre de internacionales, transfronterizos o compartidos (Autoridad Nacional del Agua, 2017).

\subsection{Desarrollo histórico: Derecho Internacional de Aguas}

En vista a esta realidad material respecto a la existencia de aguas internacionales entre Estados, se hizo necesario el establecimiento de una regulación comprehensiva sobre dicha materia que ordene la utilización

de este recurso natural, evite posibles controversias entre Estados y genere una mejor gestión de este recurso en atención a los nuevos desafíos que enfrenta la comunidad internacional. En este sentido, la rama del Derecho Internacional que se ha ocupado de esta materia es el Derecho Internacional

2 A partir de la "Convención sobre el derecho de los usos de los cursos de agua internacionales para fines distintos de la navegación" de 1997. 
de Aguas (DIA) ${ }^{34}$, el cual es el ordenamiento jurídico que regula las relaciones y conductas entre Estados o entre Estados y organizaciones internacionales en la utilización de las aguas compartidas a nivel fronterizo (Autoridad Nacional del Agua, 2017). Además, idealmente se busca que, a futuro, esta regulación se desenvuelva en el contexto de una cuenca hidrográfica y busque la gestión conjunta e integrada de aquellas para su utilización sostenible y equitativa (Global Water Partnership, 2015).

A pesar de que existan posiciones encontradas respecto a la antigüedad o novedad de esta rama del Derecho Internacional (Autoridad Nacional del Agua, 2017), lo cierto es que posturas de autores como Pierre Foy, Edith Brown y Stephen C. McCaffrey son más indispensables. Ellos sitúan el desarrollo de esta rama a la par de la evolución de la organización social humana, ya que " $[\mathrm{w}]$ ater not only is essential to human life but also fuels industry and facilitates commerce (...). [Furthermore] [i]t is well known that rivers nourished the great ancient civilizations and drove their economies" (McCaffrey, 2010, p. 58).

En esta línea, con el propósito de entender la evolución del DIA, Edith Brown realizó un trabajo compilatorio de más de 2,000 acuerdos con carácter vinculante en materia de aguas a lo largo de la historia. Se tomaron en cuenta los acuerdos a partir de $1648^{5}$. Además, tomó en cuenta 16 acuerdos

3 Se diferencia del Derecho del Mar, el cual se encuentra principalmente regido por la Convención de las Naciones Unidas sobre el Derecho del Mar (CONVEMAR) de 1982. Esta versa sobre el espacio oceánico y su utilización en todos sus aspectos, a saber, navegación, sobrevuelo, exploración y explotación de recursos, conservación, contaminación y pesca (ver información sobre el Derecho del mar en el portal Naciones UnidasCentro de Información: http://www.cinu.org.mx/temas/Derint/dermar.htm).

4 Cabe señalar que el Perú no es parte de la CONVEMAR.

5 Año que se asume como el inicio del Derecho Internacional moderno con la firma de los tratados de Westfalia. 


\section{SOBRE LA CONVENIENCIA DE LA ADHESIÓN DEL PERÚ A LA CONVENCIÓN SOBRE EL DERECHO DE LOS USOS DE LOS CURSOS DE AGUA INTERNACIONALES PARA FINES DISTINTOS DE LA NAVEGACIÓN}

listados en la Organización de las Naciones Unidas para la Alimentación y la Agricultura (en adelante, FAO) anteriores a ese año (2009, p. 232).

Es importante considerar que las aguas internacionales pueden servir para más de una función o propósito. En este sentido, estas se pueden utilizar para lo siguiente: (a) trazar las fronteras internacionales de los Estados; (b) definir la regulación de la navegación (como vías de comunicación y comercio); (c) usarla para otros usos distintos a la navegación como la agricultura, pesca, producción de energía hidroeléctrica, suministro de agua, entre otros (Caflisch, 1998). ${ }^{6}$

Estos diversos propósitos coinciden con el desarrollo histórico del DIA. Así, Brown nos presenta las tendencias históricas globales de dichos acuerdos en materia de agua. Por un lado, "boundary demarcation as the primary issue [in international water agreements] was highest during the $1700-1930$ period (...) [in the same way,] agreements that dealt primarily with navigation peaked in the [same] period" (2009, p. 235). Este escenario se debió a que, usualmente, los tratados de paz y de establecimiento de nuevos Estados contenían previsiones respecto a delimitación de fronteras y navegación. Por otro lado, "[t] hose treaties concerned primarily with allocation and use issues were most significant as a percentage of total agreements negotiated during the period 1931-2000 (...) [whereas] [a]greements concerned primarily with pollution and with ecosystem protection emerged mainly after 1950" (2009, p. 235).

De esta forma, la regulación originaria respecto a las aguas ha estado centrada en la navegación ${ }^{7}$ y la delimitación de fronteras, dada la gran importancia de estas rutas para la expansión comercial y colonial, entre otras

6 Por su parte, en su trabajo compilatorio, Brown señala que "[w]e have distinguished five major purposes of international fresh water agreements: to demarcate a boundary; to facilitate navigation; to allocate the use of water or to develop/control it for certain purposes; to protect the ecosystem; and to control pollution” (2009, p. 234).

7 Inclusive se hacía alusión a un Derecho Internacional fluvial clásico (Pastor Ridruejo, 2003). 
(Autoridad Nacional del Agua, 2017). No obstante, como se ha observado, esta rama del Derecho Internacional ha ido abarcando mayores dimensiones normativas, diferentes a su enfoque tradicional. Entre ellas se cuentan los otros usos distintos a la navegación, la protección del medioambiente y el control de la contaminación.

Sobre el particular, es importante señalar que uno de los factores que permitió la cobertura de nuevas dimensiones fue la evolución de las teorías jurídicas en torno a la tensión que subyace en esta materia entre la soberanía de los Estados y los límites que impone el Derecho Internacional. En este sentido, a continuación, realizaremos una breve revisión de las teorías mencionadas.

En primer lugar, está la teoría de la soberanía absoluta del Estado ribereño, la cual tiene su principal manifestación en la doctrina Harmon. Debido a esta última, en 1896, el fiscal general de los Estados Unidos, ante el perjuicio ocasionado a México por desviar las aguas compartidas del Río Grande, afirmó la soberanía absoluta de cada Estado al interior de su territorio sin que esto suponga un compromiso por las repercusiones fuera de sus fronteras (Autoridad Nacional del Agua, 2017). Como se observa, dicha teoría equiparaba la realidad fluyente y cíclica de las aguas internacionales a un territorio estático.

En segundo lugar, se encuentra la teoría de la integridad territorial absoluta, sostenida por Fauchille y Oppenheim, la cual sostiene que los Estados que comparten el curso de agua tienen derecho a un flujo permanente de este. En virtud de ello, ningún Estado podrá realizar acciones que afecten dicho flujo, y de esta forma, el Estado afectado tendría el derecho de demandar respecto a la continuación del flujo permanente (Autoridad Nacional del Agua, 2017). Algunos sostienen, como defecto, que esta teoría llevada al extremo podría suponer la configuración de un derecho de veto (Pastor Ridruejo, 1986). 


\section{SOBRE LA CONVENIENCIA DE LA ADHESIÓN DEL PERÚ A LA CONVENCIÓN SOBRE EL DERECHO DE LOS USOS DE LOS CURSOS DE AGUA INTERNACIONALES PARA FINES DISTINTOS DE LA NAVEGACIÓN}

En tercer lugar, cabe destacar la teoría del uso equitativo, la cual se basa en la igualdad de derechos y la soberanía compartida de los Estados. En ese contexto, postula que los cursos de agua internacionales constituyen recursos naturales compartidos, lo que implica un deber de uso equitativo de los mismos (Autoridad Nacional del Agua, 2017). A esta teoría también, en términos de Pastor Ridruejo, se la conoce como "teoría de la soberanía territorial limitada de los Estados ribereños" (2003, p. 442), por la cual el Estado ribereño puede utilizar la parte del curso de agua dentro de su territorio, en tanto ese uso no afecte a los otros Estados rivereños.

Adicionalmente, cabe señalar que existe un enfoque que es complementario a esta última teoría, denominado gestión conjunta o comunidad de intereses. Esta teoría busca ir más allá del uso equitativo y razonable, en este sentido, concibe una regulación conjunta que tiene como objeto a la cuenca hidrográfica. Por ello, plantea su regulación de forma integral como una unidad económica, social, cultural y ambiental, dejando de tomar en cuenta las fronteras entre Estados (Autoridad Nacional del Agua, 2017, p. 33).

\subsection{Los usos distintos a la navegación: La Convención de Nueva York}

Para efectos de esta investigación, nos centraremos en el análisis de los usos distintos a la navegación descritos anteriormente, cuya regulación fue producto de la evolución de las nuevas necesidades plasmadas en tratados sobre la materia. Cabe decir que dichos tratados encontraron sustento en el progreso teórico respecto a la tensión entre soberanía y el Derecho Internacional, en especial, en la teoría del uso equitativo. Esta última será una de las bases de la regulación de los usos distintos a la navegación, como veremos posteriormente.

La historia del Derecho Internacional de Aguas está basado en la celebración de tratados bilaterales, que tuvieron sus efectos limitados solo entre 
los Estados partes de dichos acuerdos. Si bien estos pueden ir formando evidencia de la práctica de los Estados en esta materia de estudio, era imprescindible contar con un tratado multilateral que supusiese una regulación de carácter general y geográficamente universal. Asimismo, debía brindar seguridad jurídica y prevenga el surgimiento de controversias internacionales.

En este sentido, dos respetables sociedades científicas —organizaciones no gubernamentales internacionales - sentaron los precedentes de los posteriores esfuerzos de codificación a nivel global de los usos no navegables de los cursos de agua internacionales. Se trata, precisamente, del Instituto de Derecho Internacional (Institut de droit international) ${ }^{8}$ y la Asociación de Derecho Internacional (International Law Association) ${ }^{9}$. Por un lado, el IDI contribuyó con lo siguiente:

(...) three resolutions concerning shared waters resources: the 1911 Madrid Resolution on International Regulations regarding the Use of International Watercourses, the 1961 Salzburg Resolution on the Use of International NonMaritime Waters and the 1979 Athens Resolution on the Pollution of Rivers and Lakes and International Law. (McCaffrey, 2010, p. 378)

Estas resoluciones contemplan las formulaciones iniciales de los conceptos que conocemos hoy en el DIA respecto a los usos no navegables. Entre otros, se hace referencia a la no realización de modificaciones en detrimento de otro Estado del curso de agua. Asimismo, consideró el

8 En adelante, IDI.

9 En adelante, ILA. 


\section{SOBRE LA CONVENIENCIA DE LA ADHESIÓN DEL PERÚ A LA CONVENCIÓN SOBRE EL DERECHO DE LOS USOS DE LOS CURSOS DE AGUA INTERNACIONALES PARA FINES DISTINTOS DE LA NAVEGACIÓN}

principio de la equidad en la solución de controversias, a tomar en cuenta las necesidades particulares de Estados, entre otros (McCaffrey, 2010).

Por otro lado, uno de los más resaltantes instrumentos desarrollado en la ILA fue el conjunto de artículos denominados las Reglas de Helsinki sobre los usos de las aguas de los ríos internacionales (en adelante, Reglas de Helsinki) de 1966. De esta forma, "[t]he Helsinki Rules represent a pioneering effort at comprehensive codification of the law of international watercourses" (McCaffrey, 2010, p. 380). En efecto, las Reglas de Helsinki constituyen un valioso articulado que tiene como base el principio de utilización equitativa y razonable. Desde esta época se va recogiendo los factores para determinar el contenido de ese principio, y se deja en claro que no existe prioridad entre los diversos tipos de usos, entre otros (McCaffrey, 2010).

No obstante, a pesar del entrañable aporte de estos instrumentos, estos no tienen carácter vinculante. Por lo tanto, su incumplimiento no generaría responsabilidad internacional ${ }^{10}$. Bajo este panorama cabe considerar lo que la doctrina señala respecto de las Reglas de Helsinki:

[d] espite their soundness, the Helsinki Rules as such had no official standing. In addition, the world's water resources, especially those of developing countries, began to be in increasingly short supply, [and due to other needs,] [t]his is why the General Assembly of the United Nations decided, in 1970, to request the [International Law Commission] ILC to prepare a set of draft articles to govern the nonnavigational uses of IWC. (Caflisch, 1998, p. 9)

10 Salvo en el caso que ciertas disposiciones reflejen la costumbre internacional. 
Sin embargo, esto no debería llevar a considerar que las Reglas de Helsinki no tuvieron ninguna utilidad. Por el contrario, no dejaron de tener influencia para identificar la necesidad de que se estudiara esta materia por la Comisión de Derecho Internacional. En tal sentido, la doctrina señala lo siguiente:

[i]t was in fact Judge E.J. Manner, the Finnish Chair of the [International Law Association] ILA Committee that prepared the [Helsinki] Rules who (...) proposed in the UN General Assembly that the ILC take up the study of the law of the non-navigational uses of international watercourses, and that it consider using the Helsinki Rules as a model for its work. (McCaffrey, 2010, p. 381)

\subsection{Creación de la Convención de Nueva York en la ONU: Comisión y Asamblea}

En respuesta a la Resolución 2669 (XXV) de la Asamblea General de las Naciones Unidas ${ }^{11}$, del 8 de diciembre de 1970, relativa al desarrollo progresivo y codificación de las normas de Derecho Internacional sobre los Cursos de Agua Internacionales, la Comisión de Derecho Internacional ${ }^{12}$ incluyó el tema "The law of the non-navigational uses of international watercourses" en su programa de trabajo durante su vigésimo tercer período de sesiones en 1971 (International Law Commission, 1996, p. 88).

11 En adelante, la AGNU.

12 En adelante, la CDI. 


\section{SOBRE LA CONVENIENCIA DE LA ADHESIÓN DEL PERÚ A LA CONVENCIÓN SOBRE EL DERECHO DE LOS USOS DE LOS CURSOS DE AGUA INTERNACIONALES PARA FINES DISTINTOS DE LA NAVEGACIÓN}

La CDI estudió la mencionada materia por un periodo de 20 años, desde 1974 hasta 1994, en los que participaron cinco Relatores Especiales ${ }^{13}$ sucesivos. Dicho trabajo estuvo influenciado, en gran proporción, por la práctica de los Estados. Esto se pudo observar de la serie de textos legales y disposiciones de tratados analizados en la materia. De este modo, cabe apreciar que, en 1991, la CDI llegó a los siguientes avances:

(...) the Commission forwarded a first draft of 32 articles to the Assembly for discussion [and commentary] by its Sixth (legal) Committee (...), [t] hereupon the [Commission] slightly amended its first draft (...). The amended text went back to the Sixth Committee. (Caflisch, 1998, p. 9)

Como la reacción de los Estados hacia la nueva versión del proyecto era favorable, el Sexto Comité "decided to forego a diplomatic conference and to have the text finalized, in the form of a multilateral treaty of codification and of progressive development of international law" (Caflisch, 1998, p. 9). Efectivamente, la Convención fue finalmente negociada entre 1996 y 1997 en el Sexto Comité (Jurídico) de la AGNU, convocado para este propósito como un "plenary ad hoc working group of the whole" (Caflisch, 1998, p. 9), sobre la base del proyecto (Draft) de artículos aprobado por la CDI (McCaffrey \& Sinjela, 1998).

Aunque la negociación no fue sencilla ${ }^{14}$, finalmente el 21 de mayo de 1997 fue adoptada por la AGNU la Convención sobre el Derecho de

13 Se trata de Richard D. Kearney, Stephen M. Schwebel, Jens Evensen, Stephen C. McCaffrey y Mr. Robert Rosenstock.

14 En especial, respecto al principio de utilización equitativa y razonable y al principio de no causar daños. 
los Usos de los Cursos de Agua Internacionales para Fines distintos de la Navegación, más conocida como la Convención de Nueva York ${ }^{15}$ (Autoridad Nacional del Agua, 2017, p. 21). Esta Convención tiene 37 artículos y está dividido en siete partes (McCaffrey \& Sinjela, 1998). Posteriormente, la UNWC entró en vigor general el 17 de agosto de 2014.

La UNCW representa un avance significativo en la codificación del Derecho Internacional de las Aguas. Esta afirmación se sustenta en el hecho que la citada Convención recoge y sistematiza los principios, derechos, obligaciones y normas procedimentales que deberán seguir los Estados que compartan un curso de agua respecto a los usos distintos a la navegación. De esta manera, promueve un marco general de cooperación estatal, adaptable a las particularidades de los diversos cursos de agua, lo cual puede realizarse a través de la suscripción de acuerdos específicos (Autoridad Nacional del Agua, 2017).

Si bien en materia de usos distintos a la navegación algunos de los instrumentos predecesores mencionados anteriormente, así como otros acuerdos de carácter regional, incluyen ciertas disposiciones sobre dichos usos, no existe un acuerdo marco especializado en esta materia tan completo a nivel global como la UNWC. En palabras de Edith Brown, “(...) is the first and only treaty that is global in geographical reach. It is also the most comprehensive in scope. In many respects it resembles a framework convention, common in international environmental law agreements" (2009, p. 257). En consecuencia, puede ser considerado como una manifestación de un tratado ley.

La gran trascendencia de la UNWC respecto a la regulación de los usos distintos a la navegación es de amplio conocimiento tanto en el entorno académico como en la aplicación práctica de estas disposiciones, usándolas como modelo de otros acuerdos regionales. En las próximas

${ }^{15}$ En adelante, la UNWC o la Convención. 


\section{SOBRE LA CONVENIENCIA DE LA ADHESIÓN DEL PERÚ A LA CONVENCIÓN SOBRE EL DERECHO DE LOS USOS DE LOS CURSOS DE AGUA INTERNACIONALES PARA FINES DISTINTOS DE LA NAVEGACIÓN}

secciones, realizaremos el análisis de esta Convención. De igual manera, se revisará la relevancia de su adhesión por el Perú.

\section{Análisis Jurídico y Contenido de la Convención}

\subsection{Naturaleza jurídica de la Convención de Nueva York en el Derecho Internacional Público}

Las normas del Derecho Internacional que conforman este sistema jurídico surgen de diversas fuentes. Así, el artículo 38 del Estatuto de la Corte Internacional de Justicia presenta una lista referencial de algunas de ellas, a saber, tratados, costumbre y principios generales. No obstante, ello no impide la existencia de otras fuentes como, por ejemplo, los actos unilaterales o las decisiones de las organizaciones internacionales ${ }^{16}$ (Daillier, Forteau, \& Pellet, 2009).

En este orden de ideas, para comenzar con el análisis de la UNWC, es importante ubicar cuál es el rol que cumple esta en el Derecho Internacional. Para ello es fundamental determinar su naturaleza jurídica. En otras palabras, ocuparse de saber qué tipo de fuente de Derecho Internacional es.

16 Las fuentes señaladas líneas arriba producen normas vinculantes del Derecho Internacional, es decir, su incumplimiento genera responsabilidad internacional. Sin embargo, en el Derecho Internacional también existe otro tipo de instrumentos considerados de carácter no vinculante, es decir, que no entrañan una directa obligatoriedad concebida de forma tradicional, pero que usualmente presentan un valioso contenido. Este último puede generar con posterioridad instrumentos vinculantes, influenciar el desarrollo de la legislación nacional y hasta generar por sí mismas un sentido de obligatoriedad entendido de forma distinta al sentido tradicional. En particular, hacemos referencia al soft law. 
En primer lugar, observamos que la Convención admite como partes tanto a Estados. Asimismo, también comprende a las organizaciones de integración económica regional, al señalar en su artículo 34 que la UNWC estará abierta a la firma de estos. Del mismo modo, en el primer párrafo de su artículo 35 establece que la mencionada Convención “(...) estará sujeta a ratificación, aceptación, aprobación o adhesión por los Estados y las organizaciones de integración económica regional” ${ }^{17}$.

En este sentido, la UNWC constituye un instrumento con vocación de establecer obligaciones internacionales hacia dos tipos de sujetos de Derecho Internacional, a saber, Estados y organizaciones internacionales. Por lo tanto, corresponde tener presente que le es aplicable, por vía consuetudinaria, las reglas consagradas en la Convención de Viena sobre el Derecho de los Tratados entre Estados y Organizaciones Internacionales, o entre Organizaciones Internacionales de $1986^{18}$. De este modo, según la definición del inciso a) del primer párrafo del artículo 2 de la CV1986, se considera que un instrumento constituye un tratado cuando este es "un acuerdo internacional regido por el derecho internacional y celebrado por escrito: i) entre uno o varios Estados y una o varias organizaciones internacionales (...)".

En este orden de ideas, la UNWC cumple con ser un acuerdo abierto a incluir tanto a Estados, como organizaciones internacionales,

17 En el inciso d), del artículo 2 de la Convención, se precisa que se entiende por organización de integración económica regional "una organización constituida por Estados soberanos de una región determinada a la cual sus Estados miembros han traspasado competencia respecto de las cuestiones regidas por la presente Convención y que ha sido debidamente autorizada de conformidad con sus procedimientos internos para firmarla, ratificarla, aceptarla, aprobarla o adherirse a ella".

18 En adelante, la CV1986. 


\section{SOBRE LA CONVENIENCIA DE LA ADHESIÓN DEL PERÚ A LA CONVENCIÓN SOBRE EL DERECHO DE LOS USOS DE LOS CURSOS DE AGUA INTERNACIONALES PARA FINES DISTINTOS DE LA NAVEGACIÓN}

como se señaló respecto de sus artículos 34 y 35. Estas últimas son denominadas "organizaciones de integración económica regional” en la referida Convención. Asimismo, un tratado es un acuerdo de voluntades de carácter internacional con intensión de vincular jurídicamente a sus partes por medio de derechos y obligaciones internacionales. Al respecto, la Convención cumple con establecer una serie de derechos y obligaciones en materia de los usos no navegables de los cursos de agua internacionales.

A modo de ejemplo, la Convención dispone en su artículo 7 la obligación de no causar daños sensibles a otros Estados del curso de agua al momento de utilizar el mismo. Por lo tanto, las partes deberán tomar todas las medidas necesarias para evitar la generación de ese daño. De igual modo, el artículo 8 contiene la obligación general de cooperar, dirigida a los Estados del curso de agua. Por su parte, el artículo 12 establece el derecho de los Estados, a los que se pueda causar un efecto perjudicial, de ser notificados oportunamente. Ello debe darse antes de la ejecución de las medidas que lo generen, incluirse en la notificación datos técnicos, resultados de una evaluación de los efectos ambientales, entre otros.

En otro extremo, la Convención cumple con ser un instrumento internacional celebrado por escrito. Sobre este punto no cabe la más mínima discusión. Cabe además considerar que sus versiones oficiales son los idiomas que Naciones Unidas emplea, lo cual se confirma en su artículo 37, que indica que el documento "original de [dicha] Convención, cuyos textos en árabe, chino, español, francés, inglés y ruso son igualmente auténticos, [fue] depositado en poder del Secretario General de las Naciones Unidas".

De igual forma, la UNWC cumple con estar regida por el Derecho Internacional, conforme a la definición de la CV1986 que comprende a Estados y organizaciones internacionales, cuyas disposiciones reflejan el derecho consuetudinario. Por lo tanto, a raíz de lo expuesto, se concluye que la naturaleza 
jurídica de la UNWC es la de un tratado, conforme a la definición contenida en el artículo 2.1 de la CV1986. En este sentido, la Convención forma parte del Derecho Internacional en calidad de tratado, por lo que tiene carácter vinculante, es decir, es de obligatorio cumplimiento.

Ahora bien, el contenido normativo que codifica la Convención es uno de carácter especializado, por lo que puede ubicarse en una de las ramas del Derecho Internacional. Así, la UNWC contiene normas del DIA que en específico buscan regular los usos de los cursos de agua internacionales para fines distintos de la navegación. De esta forma, a continuación, analizaremos con mayor precisión cuál es el ámbito que abarca la UNWC respecto a dichos usos. Del mismo modo, distinguiremos dicho ámbito de aplicación de otros instrumentos internacionales que también abordan en alguna medida esta materia.

\section{2 Ámbito de aplicación de la Convención}

En este orden de ideas, el artículo 1 de la Convención señala que su ámbito de aplicación serán "los usos de los cursos de agua internacionales y (...) sus aguas para fines distintos de la navegación y (...) las medidas de protección, preservación y ordenación relacionadas con los usos de esos cursos de agua y de sus aguas". Lo central es el uso para fines distintos de la navegación que se desprende del texto. Eventualmente, se abarcará usos que afecten o resulten afectados por la navegación.

Principalmente, el artículo 1 delimita el ámbito de aplicación de la Convención en dos líneas temáticas. Por un lado, enmarcándolo bajo el concepto de curso de agua y por el otro, acota la regulación de este a sus "usos distintos a la navegación", entre otros. Respecto a los "usos distintos a la navegación", tuvimos oportunidad de señalar en las primeras secciones que 


\section{SOBRE LA CONVENIENCIA DE LA ADHESIÓN DEL PERÚ A LA CONVENCIÓN SOBRE EL DERECHO DE LOS USOS DE LOS CURSOS DE AGUA INTERNACIONALES PARA FINES DISTINTOS DE LA NAVEGACIÓN}

este es uno de los diversos usos posibles de las aguas internacionales, cuya regulación pertenece a las más recientes, en comparación con la navegación. En este sentido, señalamos que esto se debe a la ampliación de dimensiones que experimentó el DIA gracias al progreso teórico, en específico, de la teoría del uso equitativo.

Asimismo, respecto al concepto de curso de agua, la Convención entiende por esta definición jurídica, en su artículo 2, inciso a), “[a] un sistema de aguas de superficie y subterráneas que, en virtud de su relación física, constituyen un conjunto unitario y normalmente fluyen a una desembocadura común”. En adición, señala en el inciso b), del mismo artículo, que estos cursos de agua serán considerados internacionales en tanto algunas de "[sus] partes se encuentr[en] en Estados distintos".

En este sentido, cabe precisar este último concepto en contraste con otros conceptos similares que circunscriben el ámbito de otros principales instrumentos internacionales globales en la materia. Gracias a ello, se comprenderá de manera más completa y clara el ámbito de aplicación de la UNWC y su relación con dichos instrumentos. Así, en primer lugar, las Reglas de Helsinki nos presentan, en la Regla 2, el concepto de cuenca hidrográfica internacional, la cual se define como "Zona geográfica que se extiende por el territorio de dos o más Estados y está demarcada por la línea divisoria de un sistema hidrográfico de aguas superficiales y freáticas que fluyen hacia una salida común”.

En segundo lugar, está el Convenio sobre la Protección y Utilización de los Cursos de Agua Transfronterizos y de los Lagos Internacionales de la Comisión Económica para Europa de las Naciones Unidas (o Convención de Helsinki) ${ }^{19}$ de 1992. En su artículo 1, entiende por aguas transfronterizas

${ }^{19}$ En adelante, CEPE. 
a aquellas “(...) superficiales o freáticas que señalan, atraviesan o se encuentran situadas en las fronteras entre dos o más Estados”. La definición señalada aquí resulta ser más abarcadora que la de la UNWC.

Como se observa, estos dos instrumentos hacen referencia a un concepto más amplio que el de curso de agua de la UNWC. Las Reglas de Helsinki no solamente cubren el agua que fluye sino también el conjunto de la zona geográfica. Además, guardan consistencia con la realidad hidrológica al mencionar al sistema hidrográfico en su conjunto (McCaffrey, 2010). Mientras, la CEPE, a diferencia de la UNWC, comprende todos los acuíferos, incluyendo también los acuíferos fósiles ${ }^{20}$, por lo que no está circunscrita solamente a los acuíferos relacionados con las aguas superficiales (McCaffrey, 2016).

Las Reglas de Helsinki regulan las cuencas hidrográficas internacionales (Autoridad Nacional del Agua, 2017). En este sentido, se entiende que la diferencia entre los conceptos de cuenca hidrográfica y curso de agua de estos instrumentos es el siguiente:

(...) que la cuenca hidrográfica internacional es un concepto integrador que se define como un área geográfica y, por tanto, más holístico que el de curso de agua internacional, ya que aquél abarca a otros recursos naturales asociados a dicha geografía. El concepto de curso de agua internacional, en cambio, es más limitado, puesto que solamente incluye a las aguas superficiales y a las subterráneas que son tributarias de aquellas, todas las cuales se definen como un sistema de aguas. (Global Water Partnership, 2015, p. 33)

${ }^{20}$ Aquellos que no están conectados con la superficie. 


\section{SOBRE LA CONVENIENCIA DE LA ADHESIÓN DEL PERÚ A LA CONVENCIÓN SOBRE EL DERECHO DE LOS USOS DE LOS CURSOS DE AGUA INTERNACIONALES PARA FINES DISTINTOS DE LA NAVEGACIÓN}

Al respecto, es importante señalar que el empleo del concepto cuenca hidrográfica, si bien acorde a la realidad hidrográfica, no fue bien recibido por los Estados para ser usado en un instrumento vinculante. Ello se debió a que temían que la utilización de aquel concepto regularía no solo el sistema de agua fluyente, sino también el territorio encerrado entre las aguas. Por lo tanto, para lograr la aprobación y adopción de la UNWC por los Estados, este concepto no llegó a ser incluido en este tratado (Autoridad Nacional del Agua, 2017).

Sobre el particular, cabe advertir que las Reglas de Helsinki no constituyen un instrumento vinculante, es decir, cuyo incumplimiento genere responsabilidad internacional, mientras que la UNWC, sí lo es. Esto nos recuerda uno de los obstáculos de las citadas Reglas y la relevancia de la UNWC. A pesar de ser un ámbito de aplicación un poco más restringido en cuanto a un aspecto debatible, la UNWC coadyuva a la ejecución de las Reglas de Helsinki en cierto extremo. La razón es que estas constituyeron una de las principales, sino la principal, base predecesora de la UNWC, por lo que varias de sus disposiciones y principios básicos están contenidos en ella.

De esta forma, la UNWC convierte en normas de obligatorio cumplimiento a varias disposiciones de las Reglas de Helsinki. Así también, las completa con el trabajo de profundización realizado por la CDI. Como resultado, ambos instrumentos son compatibles uno con otro.

Por otra parte, respecto a la CEPE, cabe destacar el prolongado carácter regional que mantuvo este instrumento. En este sentido, el ámbito de aplicación geográfico difiere entre la CEPE y la UNWC. Sobre el particular, la doctrina afirma lo siguiente:

[ $t$ ] he geographical scope of the two agreements was originally quite different. The UN Watercourses Convention and [its 
Draft] (...) on which it was based were always envisaged as having universal application while the UNECE Water Convention was originally designed with the ECE region in mind and until 2013 was open only to ECE member States. (McCaffrey, 2016, p. 36)

Ambos tratados constituyen entrañables y meticulosos esfuerzos en la materia, por lo que se encontrarán valiosos aportes en ambos, algunos de ellos similares, mientras que otros innovadores, propios de cada Convención. Así, por ejemplo, si bien la CEPE se caracteriza por contener disposiciones más detalladas y prever varios órganos que faciliten su implementación, la UNWC presenta, por su parte, otras ventajas en comparación con otra Convención. De igual modo, el tratamiento exhaustivo de la notificación previa de las medidas proyectadas, la que no tiene desarrollo en la CEPE, y la existencia de un mecanismo de solución de controversias necesario, y no voluntario como en el otro tratado (McCaffrey, 2016).

Asimismo, otra de las ventajas de la UNWC es el completo y minucioso desarrollo de la obligación (y principio) de utilización equitativa y razonable en los artículos 5 y 6 , concepto que constituye la piedra angular de esta área del Derecho. Este factor es una ventaja cualitativa del UNWC respecto del CEPE. Sobre este punto, la doctrina señala lo siguiente:

By contrast, the only mention of this obligation in the UNECE Water Convention is in Article 2(2)(c) (...) [its] text would seem to indicate that the obligation of equitable and reasonable utilisation only applies where there are "activities which cause or are likely to cause transboundary impact”. (...) [Also, it] could be taken to suggest that 


\section{SOBRE LA CONVENIENCIA DE LA ADHESIÓN DEL PERÚ A LA CONVENCIÓN SOBRE EL DERECHO DE LOS USOS DE LOS CURSOS DE AGUA INTERNACIONALES PARA FINES DISTINTOS DE LA NAVEGACIÓN}

the obligation of equitable and reasonable utilisation is subordinate to that of avoidance of transboundary impact. (McCaffrey, 2016, p. 37)

El contenido de ambos tratados evidencia un ámbito de aplicación en cierto extremo similar y en otro diferente. No obstante, como señala McCaffrey, no cabe duda que ambas son compatibles y "(..) are complementary and mutually supportive" (2016, p. 39). Tal es así que ello explicaría que varios Estados sean partes en ambas convenciones y se recomiende la adhesión a ambas, en la medida de lo posible (McCaffrey, 2016).

Por otra parte, tanto las Reglas de Helsinki como la CEPE no constituyen un instrumento especializado en los usos distintos a la navegación, como sí lo es la UNWC. En este sentido, las primeras abordan otros usos como la navegación, regulación sobre contaminación, el estado de las aguas subterráneas, entre otros (McCaffrey, 2010). Mientras, la CEPE surgió para ocuparse principalmente de la protección del medio ambiente y la prevención de la contaminación (McCaffrey, 2016).

En conclusión, por las razones expuestas a lo largo de la presente sección, la UNWC debe considerarse el instrumento internacional más importante en la materia de usos no navegables de aguas transfronterizas (Autoridad Nacional del Agua, 2017). Además, esta tiene carácter vinculante, es decir, es de obligatorio cumplimiento, y tiene una relación de complementariedad tanto con las Reglas de Helsinki como con la Convención del mismo nombre. A continuación, examinaremos con mayor minuciosidad el contenido de la UNWC, los principios cuyo contenido genera e influye al resto del articulado, así como las disposiciones específicas de dicho instrumento jurídico. 


\section{Principios Generales del Derecho Internacional de Aguas en la Convención}

Para comprender mejor el contenido dela UNWC es necesario conocer primero los principios a partir de los cuales se inspira el resto del articulado del mencionado tratado y desde los que se desprende su sentido lógico en conjunto. En consecuencia, en este apartado, se hará mención a tales principios. Asimismo, cabe resaltar que la trascendencia de la Convención recae también en que esta contiene, desarrolla y convencionaliza dichos principios que corresponden al DIA, por lo cual, constituye un gran aporte para esa rama del Derecho Internacional.

La UNWC comprende cinco principios expresos del DIA en la Parte II de su texto con el título de "Principios Generales". En ese sentido, a continuación, se realizará una descripción de los mismos.

\section{a) La utilización y participación equitativas y razonables (artículos 5 y 6)}

Este principio constituye la piedra angular de esta rama del Derecho Internacional (McCaffrey, 2010) y "[was] [b]orn of the United State Supreme Court's decisions in interstate apportionment cases beginning in the early twentieth century, and [was] supported by the decisions in other federal states" (McCaffrey, 2010, p. 384). Asimismo, fue aplicado a nivel internacional en anteriores ocasiones como principio de las Reglas de Helsinki (McCaffrey, 2010).

De esta manera, el principio bajo análisis se fue formando en casos como New Jersey v New York, Nebraska v Wyoming, entre otros (McCaffrey, 2010). A modo ilustrativo, en este último caso, Justice Holmes reconoce los intereses de ambos Estados y propone el reparto equitativo 


\section{SOBRE LA CONVENIENCIA DE LA ADHESIÓN DEL PERÚ A LA CONVENCIÓN SOBRE EL DERECHO DE LOS USOS DE LOS CURSOS DE AGUA INTERNACIONALES PARA FINES DISTINTOS DE LA NAVEGACIÓN}

de las aguas del río Platte del norte (McCaffrey, 2010). El mencionado principio se consolidó en la UNWC, en su artículo 5. De ese modo, el referido artículo indica que los Estados del curso de agua "utilizarán [dicho curso] en sus territorios respectivos de manera equitativa y razonable. En particular, (...) con el propósito de lograr la utilización óptima y sostenible y el disfrute máximo compatibles con la protección adecuada del curso de agua (...)”. De igual forma, añade en su segundo párrafo:

(...) participarán en el uso, aprovechamiento y protección de un curso de agua internacional de manera equitativa y razonable. Esa participación incluye tanto el derecho de utilizar el curso (...) como la obligación de cooperar en su protección y aprovechamiento (...).

Sobre el particular, el proyecto de los artículos de la Convención elaborado por la CDI en 1994, denominado Draft articles on the law of the non-navigational uses of international watercourses (en adelante, el Draft), presenta los comentarios de dicha institución sobre el contenido de cada artículo de la Convención. De este modo, respecto a la discusión sobre el concepto de equitable utilization, el Draft hace manifesto que "the principle of the sovereign equality of States results in every watercourse State having rights to the use of the watercourse that are qualitatively equal to, and correlative with, those of the other watercourse States" (International Law Commission, 1994 (46th session), p. 98). Además, agrega que " $[\mathrm{t}]$ his fundamental principle of 'equality of right' does not, however, mean that (...) the water itself is divided into identical portions. Rather, each watercourse State is entitled to use and benefit from the watercourse in an equitable manner". 
En ese sentido, "[t]he scope of a State's rights of equitable utilization depends on the facts and circumstances of each individual case, and specifically on a weighing of all relevant factors, as provided in article 6” (International Law Commission, 1994, p. 98).

En efecto, los factores y circunstancias para determinar la utilización equitativa y razonable se encuentran en el artículo 6. Este artículo presenta una lista no taxativa de siete factores a tener en cuenta (McCaffrey \& Sinjela, 1998). Principalmente, dichos factores se relaciones con aspectos geográficos, ecológicos, sociales, de necesidad económica, respecto a la población dependiente del curso de agua, efectos producidos por los usos, conservación, existencia de alternativas comparables al uso, entre otros factores que pueden no encontrarse listados. De igual modo, según McCaffrey, el listado del artículo 6 es solamente indicativo, por ello, según el análisis del caso en concreto, los factores no listados pueden ser relevantes, mientras que los que se encuentran listados pueden no serlo en gran medida

Como señala el Draft, se deberá realizar un balance (weighing), utilizando estos factores. Asimismo, como señala el artículo 6, "el peso que se le asigne a cada factor dependerá de su importancia en comparación con la de otros factores pertinentes", en el caso en concreto a analizar. De igual modo, es fundamental destacar que este principio no es abstracto ni estático, sino que

[t]he obligation of equitable and reasonable utilization is thus best understood as a process. A state's fulfillment of this obligation is dependent upon the regular receipt from other states of data and information concerning the watercourse [article 9], its provision of prior notification 


\section{SOBRE LA CONVENIENCIA DE LA ADHESIÓN DEL PERÚ A LA CONVENCIÓN SOBRE EL DERECHO DE LOS USOS DE LOS CURSOS DE AGUA INTERNACIONALES PARA FINES DISTINTOS DE LA NAVEGACIÓN}

to other states of new uses that might affect them [Part III], and its conducting TEIAs [environmental impact assessment] to determine when activities in its territory might adversely affect other states utilization of the watercourse [article 12]. (McCaffrey, 2010, p. 403)

Por lo tanto, la concretización del principio de utilización equitativa y razonable, entendido como un proceso constante, se realiza necesariamente mediante el resto de artículos que contiene la Convención. En particular, lo hace por medio de los mencionados artículos 9 y 12, entre otros. Lo cual evidencia la relevancia de este principio para el resto del articulado de la Convención.

En adición, respecto a la jurisprudencia internacional, cabe destacar el caso Gabčíkovo-Nagymaros (Hungría contra Eslovaquia), sentencia de la Corte Internacional de Justicia (en adelante, la CIJ), del 25 de septiembre de 1997. En este caso, la CIJ interpretó que la desviación de las aguas del río Danubio por Checoslovaquia significaba una medida desproporcionada en atención al principio de utilización equitativa y razonable (Global Water Partnership, 2015).

Por otra parte, respecto al segundo párrafo del artículo 5 , es relevante señalar que este enuncia un nuevo concepto, el de participación equitativa. Sobre el particular, "[t]he basic idea behind this concept is that in order to achieve the goals laid down in paragraph 1 of the article, riparian states must often cooperate with each other by taking affirmative steps (...)" (McCaffrey, 2010, p. 363). Este concepto referente a la cooperación no había sido anteriormente codificado. 


\section{b) La obligación de no causar daños sensibles (artículo 7)}

En base a este artículo se entiende que los Estados del curso de agua, "al utilizar un curso de agua (...) en sus territorios, adoptarán todas las medidas apropiadas para impedir que se causen daños sensibles a otros Estados del curso (...)”. Asimismo, cuando a pesar de ello:

[se] causen daños sensibles a otro Estado del curso (...) el Estado cuyo uso los cause deberá, a falta de acuerdo con respecto a ese uso, adoptar todas las medidas apropiadas, teniendo (...) en cuenta lo dispuesto en los artículos 5 y 6 y en consulta con el Estado afectado, para eliminar o mitigar esos daños y, cuando proceda, examinar la cuestión de la indemnización.

La relación de este concepto con el de utilización equitativa y razonable fue una de las mayores discusiones durante la negociación de la UNWC. ¿Sería este concepto uno de los factores del artículo 6 para determinar el concepto de utilización equitativa y razonable o, por el contrario, este constituiría un concepto independiente que pudiera entrar en colisión con la utilización equitativa y razonable? (McCaffrey, 2010). Al respecto, McCaffrey concluyó:

(...) the available authorities indicate that while the no-harm principle does qualify as an independent norm, it neither embodies an absolute standard nor supersedes the principle of equitable utilization where the two appear to conflict with each other. Instead, (...) it plays a complementary role, triggering 


\section{SOBRE LA CONVENIENCIA DE LA ADHESIÓN DEL PERÚ A LA CONVENCIÓN SOBRE EL DERECHO DE LOS USOS DE LOS CURSOS DE AGUA INTERNACIONALES PARA FINES DISTINTOS DE LA NAVEGACIÓN}

discussions between the states concerned and perhaps, in effect, proscribing certain forms of serious harm. [In this sense], (...) in the field of international watercourses, it is not the causing of significant harm per se, but the unreasonable causing of such harm that is prohibited (...) [i]t is thus the flexibility of the no-harm rule that makes it compatible (...) with the principle of equitable utilization. (McCaffrey, 2010, pp. 408; 436)

\section{c) La obligación general de cooperar (artículo 8)}

$\mathrm{Al}$ respecto, los Estados del curso de agua "cooperarán sobre la base de los principios de la igualdad soberana, la integridad territorial, el provecho mutuo y la buena fe a fin de lograr una utilización óptima y una protección adecuada de un curso de agua internacional”. De igual forma, según el segundo párrafo del artículo 8, serán los Estados mismos los encargados de determinar las modalidades y los mecanismos conjuntos que consideren convenientes para llevar a cabo y facilitar la cooperación. Según el Draft, la cooperación en esta materia es importante para "the attainment and maintenance of an equitable allocation of the uses and benefits of the watercourse and for the smooth functioning of the procedural rules contained in part three" (International Law Commission, 1994, p. 105) de la Convención.

En este sentido, de la redacción tanto del artículo 8 como del texto del Draft se hace manifiesto que la obligación general de cooperación está pensada en facilitar, además del contenido de la Convención en general, el principio sobre la utilización equitativa y razonable (artículo 5). En efecto, tal como observamos 
respecto a dicho principio, en la sección a), este constituye un proceso que se basa en la cooperación continua de los Estados partes en tareas concretas.

\section{d) El intercambio regular de datos e información (artículo 9)}

El intercambio regular de información es uno de los principios emergentes del Derecho Ambiental y del DIA. Se vuelve, así, ideal para estas ramas del Derecho, ya que permite conocer de manera previa las actividades que puedan tener algún impacto (Global Water Partnership, 2015). Según la Convención, “[d] e conformidad con el artículo 8, los Estados (...) intercambiarán regularmente los datos y la información que estén fácilmente disponibles sobre el estado del curso de agua, en particular los de carácter hidrológico, meteorológico, hidrogeológico y ecológico (...)". Asimismo, el artículo 9 se pone en el supuesto por el cual, de pedir uno de los Estados del curso de agua información o datos que no estén fácilmente disponibles, el otro Estado "hará lo posible por atender esta petición, pero podrá exigir que el Estado solicitante pague los costos razonables de la recopilación [y el procesamiento] (...)".

El artículo citado culmina señalando que los Estados del curso de agua “(...) harán lo posible por reunir y, en su caso, procesar los datos y la información de manera que se facilite su utilización por los Estados del curso de agua a los que sean comunicados". A su vez, del texto del mismo artículo 9, así como del Draft de artículos, se determina que "[t]he rules in this article thus constitute a specific application of the general obligation to cooperate laid down in article 8 (...)" (International Law Commission, 1994, p. 107). De igual modo, este principio sobre el intercambio regular de información es necesario para la aplicación del artículo 6, "which calls for watercourse States to take into account 'all relevant factors and circumstances' in implementing the obligation of equitable utilization laid down in article 5" (International Law Commission, 1994, p. 101). 


\section{SOBRE LA CONVENIENCIA DE LA ADHESIÓN DEL PERÚ A LA CONVENCIÓN SOBRE EL DERECHO DE LOS USOS DE LOS CURSOS DE AGUA INTERNACIONALES PARA FINES DISTINTOS DE LA NAVEGACIÓN}

Efectivamente, conforme a lo señalado en anteriores secciones, el principio de utilización equitativa y razonable es un proceso constante de cooperación entre los Estados que comparten el curso de agua en actividades concretas tales como el intercambio de información. En efecto, tal intercambio fomenta la cooperación entre los Estados que comparten cursos de aguas. Pero, sobre todo, se convierte en una expresión específica del principio de utilización equitativa y razonable y cuya necesidad se acentúa dado el carácter científico que supone el proceso de identificación de los diversos aspectos que suponen los cursos de aguas.

\section{e) Respecto a la relación entre las diferentes clases de usos (artículo 10)}

Según el artículo 10 de la Convención, salvo exista un acuerdo o costumbre que señale lo contrario, "ningún uso de un curso de agua internacional tiene en sí prioridad sobre otros usos". Por otra parte, en una situación de conflicto entre varios usos, este "se resolverá sobre la base de los artículos 5 a 7, teniendo especialmente en cuenta la satisfacción de las necesidades humanas vitales”. Este artículo señala el principio general por el que "no use of an international watercourse enjoys inherent priority over other uses" (International Law Commission, 1994, pp. 109-110). No obstante, en el articulado de la Convención se ha dado preferencia a un acuerdo existente o a la costumbre que pueda ser de aplicación a las particularidades del curso de agua específico, colocando a esta disposición como regla residual en favor de respetar la voluntad de las partes. 


\section{La Estructura de la Convención de Nueva York a la Luz de los Principios Esbozados}

La UNWC consta de siete partes. La parte I se denomina "Introducción" y contiene en su artículo 1 la delimitación del ámbito de aplicación de la Convención, el cual ya se desarrolló en una sección previa. En su artículo 2, comprende los términos relevantes, la mayoría de los cuales también ya fueron señalados. Asimismo, en sus artículos 3 y 4 , refiere la regulación respecto a otros acuerdos en materia de cursos de agua que pueda tener el Estado parte; desarrollo que realiza en la siguiente sección respecto a los otros instrumentos internacionales con los que cuenta el Perú.

La parte II de la UNWC se denomina "Principios generales" y comprende el artículo 5 hasta el artículo 10. Estos son los cinco principios expresos del DIA desarrollados en el apartado anterior. Dichos principios son de gran relevancia, ya que, como se observó, inspiran el resto del articulado de la Convención, en especial, el principio sobre la utilización equitativa y razonable, ubicado en los artículos 5 y 6.

De igual modo, la parte III tiene como designación "Medidas proyectadas". Respecto a estas, el artículo 11 establece la obligación general por la que "[l] os Estados del curso de agua intercambiarán información y se consultarán y, si es necesario, negociarán acerca de los posibles efectos de las medidas proyectadas sobre el estado de un curso de agua internacional". Como se observa, este artículo guarda relación con los principios esbozados en la sección anterior debido a que busca concretizarlos.

Al respecto, según el Draft, mediante los artículos 12 al 19, los cuales también conforman la "tercera parte" de la Convención, se establece un marco jurídico procesal para las medidas proyectadas que puedan causar un efecto perjudicial, el que se activa mediante el criterio "efecto perjudicial 


\section{SOBRE LA CONVENIENCIA DE LA ADHESIÓN DEL PERÚ A LA CONVENCIÓN SOBRE EL DERECHO DE LOS USOS DE LOS CURSOS DE AGUA INTERNACIONALES PARA FINES DISTINTOS DE LA NAVEGACIÓN}

sensible", denominado en inglés "significant adverse effect" (International Law Commission, 1994 (46th session), p. 111). Respecto a este criterio, cabe hacer una distinción entre el "significant adverse effect", que es un elemento central de esta tercera parte de la Convención. Asimismo, es distinto del criterio "significant harm" del principio respecto a no causar daños sensibles del artículo 7.

Según el Draft, el criterio "significant adverse effect" "is intended to be lower than that of 'significant harm' under article 7 . Thus a 'significant adverse effect' may not rise to the level of 'significant harm' within the meaning of article 7" (International Law Commission, 1994, p. 111). La lógica de su menor impacto respecto del criterio "significant harm" es que un criterio de mayor gravedad no puede operar con facilidad en la lógica de la información ágil que esta tercera parte de la Convención implica. Su empleo traería una serie de desventajas a estos artículos.

En este sentido, la presente sección de la Convención regula, en sus artículos, el procedimiento que comprende la notificación de las medidas proyectadas (artículo 12), el plazo para responder la notificación (artículo 13) y las obligaciones del Estado notificante durante ese plazo (artículo 14). También contempla la respuesta a la notificación o la falta de esta (artículos 15 y 16), las consultas y negociaciones sobre las medidas (artículo 17), así como un procedimiento aplicable a la falta de notificación (artículo 18). Por último, observa la ejecución urgente de las medidas proyectadas (artículo 19).

Por otro lado, la parte IV de la Convención trata en mayor detalle la "Protección, preservación y gestión" de los cursos de agua. Según el Draft, el artículo 20 establece la obligación general de proteger y preservar los ecosistemas de los cursos de agua (International Law Commission, 1994, p. 118). A este tenor, por el artículo 20 explica que "[l]os Estados del curso de agua protegerán y preservarán, individual y, cuando proceda, conjuntamente, los ecosistemas de [dichos] cursos de agua (...)”. 
A partir de este artículo es que "[i]n view of the general nature of the obligation contained in this article, the Commission was of the view that it should precede the other more specific articles in part four" (International Law Commission, 1994, p. 118). De esta forma, los siguientes artículos de esta sección desarrollan dicha obligación general. En este sentido, se hace referencia a la prevención, reducción y control de la contaminación (artículo 21); el impedimento de introducción de especies extrañas o nuevas (artículo 22); la protección y preservación del medio marino (artículo 23), así como la regulación en torno a la ordenación (artículo 24), regulación (artículo 25) e instalaciones (artículo 26) aplicables a esta materia.

La parte V de la Convención se refiere a las "Condiciones perjudiciales y situaciones de emergencia”. Esta sección contiene dos artículos, el primero referido a las condiciones perjudiciales (artículo 27) y el segundo respecto de las situaciones de emergencia (artículo 28). De esta manera, sobre la diferencia entre ambos artículos, el Draft señala que "[t]he measures called for in preventing and mitigating these conditions are of an anticipatory nature and are thus quite different from those involved in responding to emergencies" (International Law Commission, 1994, p. 128). Cabe resaltar que ambos artículos se aplican tanto a causas naturales como a las ocasionadas por el comportamiento humano, por ejemplo, a las crecidas, el deshielo, los desprendimientos de tierra o los accidentes industriales (International Law Commission, 1994).

Respecto a la parte VI, esta contiene "Disposiciones diversas" respecto a los cursos de agua internacionales e instalaciones en tiempo de conflicto armado (artículo 29), el cual "(...) serves as a reminder [of] (...) the (...) rules of international law applicable". Así también, respecto a los procedimientos indirectos (artículo 30), aplicables en circunstancias en las cuales "[it] may raise serious obstacles to the kinds of direct contacts provided for in articles 9 to 19” (International Law Commission, 1994 (46th session), p. 132). Igualmente, 


\section{SOBRE LA CONVENIENCIA DE LA ADHESIÓN DEL PERÚ A LA CONVENCIÓN SOBRE EL DERECHO DE LOS USOS DE LOS CURSOS DE AGUA INTERNACIONALES PARA FINES DISTINTOS DE LA NAVEGACIÓN}

respecto a los datos e información vitales para la defensa y la seguridad nacional (artículo 31) y respecto a la no discriminación (artículo 32).

Asimismo, contiene un artículo sobre solución de controversias (artículo 33). Este se lee de manera conjunta con los artículos contenidos en su apéndice, los cuales desarrollan más a fondo sus reglas procedimentales. Según menciona el Draft, dicho artículo constituye una regla residual, ya que "(...) applies where the watercourse States concerned do not have an applicable agreement for the settlement of such disputes" (International Law Commission, 1994, p. 134).

Finalmente, la parte VII contiene las "Disposiciones finales" de la Convención respecto a la firma (artículo 34), la cual se cerró el año 2000. Toma en cuenta, también, la ratificación, aceptación, aprobación o adhesión (artículo 35), por las cuales el Perú puede adherirse a la Convención. A su vez, la entrada en vigor (artículo 36), la cual se efectuó el 17 de agosto de 2014; y los textos auténticos a los que ya se hicieron referencia en otro apartado.

De esta revisión general de la Convención de Nueva York, se observa que esta contiene un completo marco jurídico muy valioso sobre la regulación de los usos distintos a la navegación de los cursos de agua internacionales. Específicamente, este gira en torno a sus principios contenidos en la Parte II de su articulado, y en especial, respecto al principio de utilización equitativa y razonable. Este último implica la realización de un balance tomando en cuenta las particularidades del curso de agua, en concreto, para respetar los derechos de cada Estado del curso.

En este sentido, la adhesión a este tratado brindaría al Estado peruano una regulación marco completa sobre la materia. La misma, comprende los aspectos más importantes del DIA y que a la vez es suficientemente flexible para analizar los casos en concreto. Con ello, dejaría abierta la posibilidad de los Estados de establecer otros acuerdos internacionales sobre la materia. 
A continuación, se profundizará en las razones por las que el Estado peruano debería adherirse a la UNWC. Por ello, para tener un panorama completo, primero se desarrollará la experiencia peruana en tratados sobre la materia. Luego de ello, se hará una conexión con los aportes que entraña la UNWC.

\section{La Conveniencia de la Adhesión del Perú a la Convención de Nueva York}

\section{1 La experiencia peruana en tratados sobre aguas}

Existen acuerdos multilaterales regionales y acuerdos bilaterales en materia de DIA en los que el Estado peruano es parte. Al respecto, existen tres áreas geográficas principales sobre las que el Perú ha tenido mayor experiencia en materia de acuerdos dirigidos a cuencas de agua específicas. Entre ellas, figuran la cuenca amazónica, la cuenca del lago Titicaca y las cuencas compartidas con Ecuador. A continuación, se hará referencia a estas tres áreas y a sus respectivos instrumentos internacionales.

En primer lugar, está el Tratado de Cooperación Amazónica, adoptado el 3 de julio de 1978. Este entró en vigor general el 2 de agosto de 1980 entre Bolivia, Brasil, Colombia, Ecuador, Guyana, Perú, Surinam y Venezuela. Dicho instrumento internacional tiene como objeto, según su preámbulo y artículo I, "promover el desarrollo armónico de sus respectivos territorios amazónicos [para lograr] (...) resultados equitativos y mutuamente provechosos, así como para la preservación del medio ambiente y la conservación y utilización racional de los recursos naturales de esos territorios”. En esta misma línea, su ámbito de aplicación, según el artículo II, son "los territorios de las Partes Contratantes en la Cuenca Amazónica, así como también (...) cualquier territorio de una Parte Contratante que, 


\section{SOBRE LA CONVENIENCIA DE LA ADHESIÓN DEL PERÚ A LA CONVENCIÓN SOBRE EL DERECHO DE LOS USOS DE LOS CURSOS DE AGUA INTERNACIONALES PARA FINES DISTINTOS DE LA NAVEGACIÓN}

por sus características geográficas, ecológicas o económicas se considere estrechamente vinculado a la misma”.

Este tratado, al igual que la UNWC, tiene como objetivo la utilización racional de los recursos hídricos, el intercambio de información, entre otros. Además, a diferencia de la citada Convención, también comprende la navegación, el transporte, las telecomunicaciones, la investigación científica, los servicios de salud, entre otros. Esta ampliación obedece a las necesidades específicas de las partes que celebran el Tratado.

En segundo lugar, respecto al lago Titicaca, es importante resaltar que este forma parte del denominado sistema TDPS. Por estas siglas, comprende al lago Titicaca, el río Desaguadero, el lago Poopó y el salar de Coipasa. Asimismo, abarca un conjunto de cuencas y subcuencas hidrográficas interconectadas ubicadas en la meseta del Collao (Autoridad Nacional del Agua, 2017).

Los antecedentes de la relación de cooperación en materia de aguas entre Bolivia y Perú datan desde hace varios años atrás. No obstante, el trabajo más importante lo constituye la creación de la Autoridad Binacional Autónoma del Sistema Hídrico del Lago Titicaca, Río Desaguadero, Lago Poopó y Salar de Coipasa. Esta surgió a partir de la IV Reunión Ordinaria de la Sub-Comisión Mixta Peruano Boliviana para el Desarrollo de la Zona de Integración del Lago Titicaca de 1992. Debido a ella, se firmó y entró en vigor, el 15 de junio de 1993, el Acuerdo para la Creación de la Autoridad Binacional Autónoma de la Cuenca del Sistema Lago Titicaca, Río Desaguadero, Lago Poopó y Salar de Coipasa (Autoridad Nacional del Agua, 2017).

En tercer lugar, hay que mencionar las principales cuencas compartidas con Ecuador. Entre ellas figuran las de los ríos PuyangoTumbes, Catamayo-Chira y Zarumilla. Cabe agregar que también se cuenta con experiencias previas, incluso anteriores a la Guerra del Cenepa de 1995. Sin embargo, son otros dos los acuerdos más relevantes sobre la materia. 
JORGE RAFFO CARBAJAL, PABLO ROSALES ZAMORA Y DIANA ADUVIRI CHOQUE

Por un lado, se encuentra el Acuerdo Amplio PeruanoEcuatoriano de Integración Fronteriza, Desarrollo y Vecindad, firmado el 26 de octubre de 1998 y en vigor desde el 13 de mayo de 1999. Su firma se dio a propósito del Acuerdo de Brasilia y el fin de la Guerra del Cenepa (Autoridad Nacional del Agua, 2017). El mencionado Acuerdo Amplio tiene como uno de sus elementos el Plan Binacional de Desarrollo de la Región Fronteriza. Con más exactitud, se hace cargo, según el artículo 20 del Acuerdo Amplio, de cuatro programas que abarcan no solamente el aprovechamiento de los recursos hídricos y los aspectos ambientales, sino que tienen un carácter amplio y comprenden otros aspectos, tales como el mejoramiento de la infraestructura y la promoción de inversión privada.

Por otro lado, el Perú firmó el 22 de octubre de 2009 el Acuerdo entre la República del Perú y la República del Ecuador para el Establecimiento de la Comisión Binacional para la Gestión Integrada de los Recursos Hídricos de la Cuenca Hidrográfica Transfronteriza del Río क Zarumilla. Este Acuerdo tiene como uno de sus objetivos principales, según su artículo 2 inciso 3, promover el "desarrollo, conservación y gestión de los recursos hídricos transfronterizos, generando un positivo impacto en la calidad de vida de la población". Si bien el Tratado conlleva esta finalidad, es preciso indicar que no ha entrado todavía en vigor.

Finalmente, en este apartado se han presentado los principales acuerdos multilaterales regionales y bilaterales en materia de protección de recursos hídricos con los que cuenta el Perú. No obstante, a pesar de la regulación que estos brindan a sus cursos de agua específicos, a saber, las aguas de la Amazonía, el sistema del lago Titicaca y la serie de cursos con el Ecuador, aquella regulación no es suficiente para contar con un marco normativo completo. En especial, debido a que el Perú cuenta con 34 


\section{SOBRE LA CONVENIENCIA DE LA ADHESIÓN DEL PERÚ A LA CONVENCIÓN SOBRE EL DERECHO DE LOS USOS DE LOS CURSOS DE AGUA INTERNACIONALES PARA FINES DISTINTOS DE LA NAVEGACIÓN}

cuencas transfronterizas compartidas con Ecuador, Colombia, Brasil, Bolivia y Chile, muchas de ellas no cuentan con regulación alguna (Autoridad Nacional del Agua, 2017).

Tal cantidad de recursos hídricos compartidos amerita una regulación marco que les brinde protección. Por tanto, debido a que la UNWC es uno de los instrumentos más importantes en la materia y cuya regulación es pilar del DIA (Autoridad Nacional del Agua, 2017), se hace evidente la contribución que significaría la adhesión al referido tratado. Además, no hay que dejar de lado el aporte que podría significar para el desarrollo de la legislación nacional sobre la materia.

\subsection{Razones jurídicas que respaldan la conveniencia de la adhesión del Perú}

Como observamos en el apartado sobre el ámbito de aplicación de la UNWC, esta constituye el principal instrumento internacional vinculante a nivel universal especializado en los usos distintos a la navegación de los cursos de agua internacionales. Por ello, no abarca conceptos más extensos que suelen implicar mayor desacuerdo entre los Estados al momento de obligarse mediante la suscripción de tratados de carácter vinculante. De esta forma, la Convención tiene un carácter complementario con otros instrumentos internacionales universales similares como lo son las Reglas de Helsinki y la Convención de Helsinki (CEPE). Respecto al primer instrumento citado, este constituye un predecesor fundamental de la UNWC, la cual se elaboró en observancia de las Reglas de Helsinki. Por lo tanto, la UNWC de cierta forma materializa y vuelve vinculantes, vía convencional, varias disposiciones de las Reglas de Helsinki, las mismas que continúan funcionando como un instrumento guía a nivel internacional. 
Por otra parte, también habíamos desarrollado la relación de la UNWC con la CEPE. Señalamos que ambas presentan entrañables esfuerzos de codificación en la materia. Aunque la CEPE no tiene un carácter tan especializado con la de Nueva York, por tanto, así como se pueden encontrar similitudes entre ambas, también se pueden encontrar disposiciones innovadoras propias de cada una.

De esta forma, hicimos mención a disposiciones de la UNWC, que no se encuentran en la otra convención (CEPE) y que respaldan la importancia de la adhesión del Perú a la UNWC. Dentro de estas se encontraban, por ejemplo, el desarrollo extenso y minucioso del principio de utilización equitativa y razonable, concepto que es elemento fundamental del Derecho de Aguas. De igual modo, figura la esquematización detallada de un mecanismo de solución de controversias, incluyendo sus reglas procedimentales, entre otros.

En este sentido, se citó la opinión general respecto a la compatibilidad, complementariedad y mutuo soporte de ambas convenciones. Además, se tomó en cuenta la existencia de varios Estados que son partes en ambas y usualmente, de ser posible, se recomienda la adhesión a ambas. Aunque un primer paso fundamental es la adhesión a la UNWC, considerada el instrumento jurídico más importante en la materia.

En sus artículos 3 y 4, la UNWC prevé la situación en la que existan otros acuerdos respecto a los cursos de agua y a la posibilidad de ser parte de dichos acuerdos. Este aspecto resulta relevante para el caso del Perú, debido a que nuestro país es parte de tratados en la materia respecto a la gestión y protección de aguas específicas en sus fronteras. De esta forma, los artículos referidos de la UNWC señalan una serie de supuestos que revelan la interrelación y funcionamiento de esta Convención con los otros acuerdos sobre cursos de agua que tiene y/o tenga el Estado peruano. 


\section{SOBRE LA CONVENIENCIA DE LA ADHESIÓN DEL PERÚ A LA CONVENCIÓN SOBRE EL DERECHO DE LOS USOS DE LOS CURSOS DE AGUA INTERNACIONALES PARA FINES DISTINTOS DE LA NAVEGACIÓN}

El inciso 1 del artículo 3, de la mencionada Convención, hace referencia al supuesto en el que ya se cuente con otro acuerdo en vigor a la fecha de hacerse parte de la Convención. Este inciso señala que "nada de lo dispuesto en [la] Convención afectará a los derechos u obligaciones [del] (...) Estado derivado de [ese] acuerdo”. Por lo tanto, los derechos y obligaciones contenidos tanto en el Tratado de Cooperación Amazónica, como en los demás instrumentos del Perú citados, quedarán resguardados.

Asimismo, el inciso 2 del artículo 3, abre la posibilidad a que los Estados partes de estos otros acuerdos " $\mathrm{p}$ [uedan] considerar, de ser necesario, si han de armonizar esos acuerdos con los principios básicos de la (...) Convención”. Este aspecto puede resultar de relevancia para el Perú, por ejemplo, en tanto varios de los otros acuerdos con los que cuenta, respecto a sus cuencas específicas, mencionan el principio de utilización equitativa. Sin embargo, este no llega a tener un contenido claro. En este sentido, la UNWC brinda las pautas para aplicar este principio en sus artículos 5 y 6 .

Los incisos 3, 4 y 5 del artículo 3 de la Convención, se refieren a la posibilidad de sus Estados partes para concertar uno o varios acuerdos nuevos que "apliquen y adapten las disposiciones de la (...) Convención a las características y usos de un determinado curso de agua internacional o de una parte de él” (inciso 3 y 5). O que, también, por propia decisión los Estados "concierten un acuerdo de curso de agua (...)", teniendo cuidado que no se menoscabe de manera sensible el uso de las aguas de otros Estados (inciso 4). En consecuencia, quedará abierta la posibilidad a futuro para el Perú de establecer nuevos acuerdos sobre zonas específicas contando con la destacable regulación de la UNWC como base.

En adición, el inciso 6 del artículo 3 se pone en el supuesto en el que algunos de los Estados limítrofes de un mismo curso de agua internacional tengan otro acuerdo diferente a la Convención. Sobre el particular indica, 
con respecto a los Estados del curso de agua que no sean parte de ese otro acuerdo, que "ninguna de las disposiciones del [otro acuerdo] afectará a los derechos u obligaciones [que tengan en virtud de la Convención]”. Por consiguiente, la UNWC brindaría un margen de protección base al Perú ante la creación de otros acuerdos regionales o bilaterales sobre sus cursos de agua internacionales de los cuales no sea Estado parte.

En concordancia con lo anterior, el artículo 4 de la Convención nos sitúa en un segundo plano de garantías brindadas por esta frente a otros acuerdos en los que el Perú no sea Estado parte y que involucre sus cursos de agua. En otras palabras, en un primer plano, mediante el inciso 6 de su artículo 3, la Convención brinda una protección base que no será afectada por otros acuerdos. Mientras, por el artículo 4, en un segundo plano, la Convención otorga facultades sobre esos otros acuerdos en los dos siguientes supuestos.

El primer supuesto de intervención en los otros acuerdos mencionados, según el inciso 1 del artículo 4 de la Convención, es en el escenario de un acuerdo que se aplique a la totalidad del curso de agua internacional. Ante esto, el artículo dispone que el "Estado del curso de agua [no incluido por el acuerdo] tiene derecho a participar en la negociación, (...) a llegar a ser parte en él, así como a participar en cualesquiera consultas sobre [dicho acuerdo]". Mientras, el segundo supuesto de intervención, retratado en el inciso 2 del artículo 4 de la Convención, se presenta si el uso de uno de los Estados del curso de agua sobre este pudiera resultar afectado de forma sensible por la ejecución de un acuerdo que no lo incluya y que se aplique solo a una parte del curso de agua. En este caso, el Estado cuyo uso resulte afectado "tendrá derecho a participar en las consultas sobre tal acuerdo y, cuando proceda, a negociar de buena fe para hacerse parte de [ese] acuerdo, en la medida en que su uso resulte afectado por [tal] acuerdo". 


\section{SOBRE LA CONVENIENCIA DE LA ADHESIÓN DEL PERÚ A LA CONVENCIÓN SOBRE EL DERECHO DE LOS USOS DE LOS CURSOS DE AGUA INTERNACIONALES PARA FINES DISTINTOS DE LA NAVEGACIÓN}

Por lo señalado líneas arriba, nada impide al Estado peruano adherirse a la UNWC. La razón está en que es compatible y complementaria tanto con otros acuerdos de carácter universal similares sobre la materia como con los demás acuerdos regionales que tiene el Perú en sus fronteras con otros Estados limítrofes. Por el contrario, la UNWC brindará un marco estable y flexible de regulación que está en concordancia con el resto del Derecho de Aguas y que puede dotar de precisión y mayores recursos a los acuerdos regionales del Perú.

\section{Reflexión Final}

Como observamos a lo largo de la presente investigación, la realidad material de la existencia de los cursos de agua entre Estados hace necesaria una regulación completa que ordene su utilización y evite posibles controversias entre ellos. En este sentido, el Perú cuenta con 34 cuencas transfronterizas con sus Estados limítrofes (Autoridad Nacional del Agua, 2017), la mayoría de los cuales no tiene una regulación marco que los proteja. Ante ello, la UNWC es ampliamente reconocida como uno de los instrumentos vinculantes más importantes en la materia. En efecto, la Convención tiene naturaleza jurídica de tratado, es decir, es de obligatorio cumplimiento. Por lo tanto, su contenido, que protegería a los cursos de agua del Perú, tiene fuerza normativa.

Asimismo, la Convención contiene uno de los desarrollos más completos respecto al principio eje del DIA: la utilización equitativa y razonable. Esto nos dotaría de un conjunto normativo internacional valioso para la protección de nuestros cursos de agua. Lo mismo ocurre respecto a los demás principios mencionados explícitamente en la Convención.

No obstante, la Convención no solo nos brindaría importantes normas generales como los principios. También contiene extensamente en su articulado las normas procedimentales que concretizarán esos principios. 
De esa forma, establece obligaciones puntuales y realizables. Dentro de estas obligaciones se encuentran, a modo ilustrativo, el intercambio de información, el procedimiento respecto de las medidas proyectadas, el procedimiento sobre la solución de controversias, la compatibilidad con otros acuerdos internacionales preexistentes del Estado, entre otros.

Por otra parte, es apreciable que la Convención sea un tratado capaz de brindar márgenes de flexibilidad. Gracias a ello, permite una mejor adecuación a las particularidades del Perú, en tanto varias de sus disposiciones se aplican de acuerdo a las características del caso concreto. Además, algunas son residuales, con lo que prevalece la voluntad de las partes.

Finalmente, por todo lo expuesto en los apartados anteriores, se concluye que la UNWC constituye un tratado universal sustancial en la protección y gestión de los usos de los cursos de agua distintos a la navegación, el cual contiene un marco jurídico completo y flexible que regula dicha materia. En este sentido, la adhesión del Estado peruano resultaría beneficioso para la protección de sus 34 cuencas transfronterizas. Por medio de ello, les brindaría una regulación internacional marco que es compatible con los demás instrumentos internacionales universales sobre la materia, así como con los instrumentos regionales suscritos por el Perú. 


\section{SOBRE LA CONVENIENCIA DE LA ADHESIÓN DEL PERÚ A LA CONVENCIÓN SOBRE EL DERECHO DE LOS USOS DE LOS CURSOS DE AGUA INTERNACIONALES PARA FINES DISTINTOS DE LA NAVEGACIÓN}

\section{Referencias}

Aguilar, G., \& Iza, A. (2009). Gobernanza de Aguas Compartidas. Aspectos Jurídicos e Institucionales. Gland, Suiza: Unión Internacional para la Conservación de la Naturaleza y de los Recursos Naturales (UICN).

Autoridad Nacional del Agua. (2017). Cursos de Agua Internacional y Cuencas Transfronterizas en la Legislación Peruana. Lima, Perú: Autoridad Nacional del Agua.

Brown, E. (2009). The Evolution of International Water Law. En Collected Courses of the Hague Academy of International Law (pp. 163-404). Boston, USA: The Hague Academy of International Law.

Caflisch, L. (1998). Regulation of the Uses of International Watercourses. En International Watercourses. Enhancing Cooperation and Managing Conflict. Proceedings of a World Bank Seminar. WTP414 (pp. 3-16). Washington, D.C., USA: The World Bank.

Daillier, P., Forteau, M., \& Pellet, A. (2009). Droit International Public. Paris, France: Librairie générale de droit et de jurisprudence.

Global Water Partnership. (2015). El derecho internacional de aguas en América Latina. Manual de capacitación. Montevideo, Uruguay: GWP.

International Law Commission. (1994). Draft articles on the law of the nonnavigational uses of international watercourses and commentaries thereto and resolution on transboundary confined groundwater $46^{\text {th }}$ session. Geneva, Switzerland: International Law Commission.

International Law Commission. (1996). Yearbook of the International Law Commission 1994, 46th session. Volume II, Part Two. Geneva, Switzerland: United Nations.

McCaffrey, S. (2010). The Law of International Watercourses. New York, USA: Oxford University Press.

McCaffrey, S. (2016). Implementation and Relationship to the UNECE Water Convention. Environmental Policy and Law, 46(1), 35-39. 
McCaffrey, S. (s. f.). International Watercourses. The United Nations Convention on the Law of the Non-Navigational Uses of International Watercourses. Audiovisual Library of International Law. Recuperado de <http://www.un.org/law/avl/>

McCaffrey, S., \& Sinjela, M. (January of 1998). The 1997 United Nations Convention on International Watercourses. The American Journal of International Law, 92(1) pp. 97-107.

Novak, F. (1994). La teoría de los actos unilaterales de los Estados. Agenda Internacional, 1(1),149-166.

Pastor Ridruejo, J. (1986). Curso de derecho internacional público. Madrid, España: Editorial Tecnos.

Pastor Ridruejo, J. (2003). Curso de derecho internacional público y organizaciones internacionales. Madrid, Espańa: Editorial Tecnos. 